\title{
HOMOTOPY CLASSIFICATION OF MAPS BY COHOMOLOGY HOMOMORPHISMS (1)
}

\author{
BY \\ EMERY THOMAS
}

1. Introduction. Let $f$ be a map from a space $X$ to a space $Y$. To what extent is the homotopy class of $f$ determined by the cohomology homomorphisms induced by it? If $X$ is a complex of dimension $n$ and $Y$ an $n$-sphere, then by the Hopf Theorem $f$ is determined up to homotopy by its induced cohomology homomorphism with integer coefficients. Another such example is given by F. Peterson [14]. Let $X$ be a complex of dimension $\leqq 2 n$ and let $\omega$ denote a complex $n$-plane bundle over $X$. Suppose that $X$ has no torsion in its even dimensional cohomology groups. Peterson shows that the bundle $\omega$ is then determined by its Chern classes. Here $Y$ is the classifying space $B_{U(n)}$, and $f$ is the characteristic map for $\omega$.

We consider arcwise connected spaces with basepoint, and denote by $[X, Y]$ the set of homotopy classes of (basepoint preserving) maps from $X$ to $Y$. Let $G$ be an abelian group and let $\bar{H}^{*}(X ; G)$ denote the reduced singular cohomology groups of $X$ with coefficients in $G$. Define a function (see [20, p. 14])

$$
[X, Y] \stackrel{\lambda_{G}}{\longrightarrow} \operatorname{Hom}\left(\bar{H}^{*}(Y ; G), \bar{H}^{*}(X ; G)\right)
$$

by setting $\lambda_{G}[f]=f^{*}$, where $[f]$ denotes the homotopy class of $f$ and $f^{*}$ its induced cohomology homomorphism. Define $N_{G}[X, Y]$ to be the kernel of $\lambda_{G}$ and set

$$
N[X, Y]=\bigcap N_{G}[X, Y]
$$

where the intersection is taken over all finitely generated abelian groups. (We will denote the category of these groups by $\mathscr{G}$.)

The purpose of this paper is to define invariants whose vanishing implies that $N[X, Y]=0$. For in this case a map is null-homotopic if, and only if, all of its induced cohomology homomorphisms (with coefficients $\in \mathscr{G}$ ) are zero.

Under the hypotheses of Theorem 1.1 below, the set $[X, Y]$ has a natural group structure (see James [9]), and one then can say more about the subset $N[X, Y]$.

THeORem 1.1. Let $X$ be a CW-complex and suppose that either $X$ is a suspension or $Y$ is a homotopy-associative $H$-space whose singular homology

Received by the editors October 26, 1962.

(1) Research supported by the Air Force Office of Scientific Research. 
groups are of finite type. Then $N[X, Y]$ is a normal subgroup of the group $[X, Y]$, and two classes $[f]$ and $[g]$ belong to the same coset of $N[X, Y]$ if, and only if, they induce identical cohomology homomorphisms, taking coefficients in all groups $G \in \mathscr{G}$.

The proof is given in an appendix $(\S 5)$.

2. The invariants. Let $Y$ be a space which is $n$-simple for all $n \geqq 1$. In particular, this means that $\pi_{1}(Y)$ is abelian. Suppose that $0<n(1)<n(2)<\cdots$ are the dimensions in which $Y$ has nonzero homotopy groups, and set $\pi_{i}=\pi_{n(i)}(Y)$. Recall that a Postnikov system for $Y$ provides a sequence of fibre spaces (and commutative diagrams)

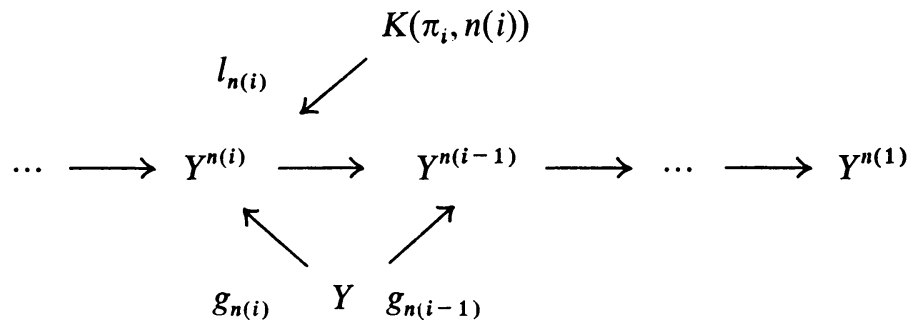

such that the map $g_{n(i)}$ induces an isomorphism on homotopy groups in dimensions $\leqq n(i)$. Denote by $k_{n(i)}$ the $i$ th Postnikov invariant of $Y$ : that is,

$$
k_{n(i)}: Y^{n(i)} \longrightarrow K\left(\pi_{i+1}, n(i+1)+1\right) .
$$

Define a sequence of first order cohomology operations (for each space $Y$ ) by

$$
\Psi_{n(i)}=-k_{n(i)} \circ l_{n(i)}: K\left(\pi_{i}, n(i)\right) \rightarrow K\left(\pi_{i+1}, n(i+1)+1\right) .
$$

Set $\Phi_{n(i)-1}=\sigma \Psi_{n(i)}$, where $\sigma$ denotes the suspension of cohomology operations. (If $n(1)=1$, then $\Phi_{0}=0$.)

We next define a sequence of non-negative integers $\tau_{n(i)}$ as follows. Suppose first that $\pi_{i}$ is a cyclic group, and let $f: S^{n(i)} \rightarrow Y$ represent a generator. Define $\tau_{n(i)}$ to be the least positive integer such that

$$
\tau_{n(i)} s_{i} \in f^{*} H^{n(i)}\left(Y ; \pi_{i}\right),
$$

where $s_{i}$ generates the cyclic group $H^{n(i)}\left(S^{n(i)} ; \pi_{i}\right)$. If $f^{*} H^{n(i)}\left(Y ; \pi_{i}\right)=0$, or if $\pi_{i}$ is not $\operatorname{cyclic}(2)$, set $\tau_{n(i)}=0$.

Denote by $\tau_{n(i)}{ }^{*}$ the cohomology operation given by multiplying each cohomology class by the integer $\tau_{n(i)}$; we consider this operation only in dimension $n(i)$, with coefficients in $\pi_{i}$. Thus with each space $Y$ we associate three sequences of cohomology operations: $\Psi_{n(i)}, \Phi_{n(i)-1}$, and $\tau_{n(i)} *$.

${ }^{2}$ ) The definition of the invariants $\tau_{n(i)}$ can be extended to the case that $\pi_{i}$ is not cyclic. Then $\tau_{n(i)}$ becomes a set of elements from Hom $\left(\pi_{i}, \pi_{i}\right)$, but the usefulness of the generalization to applications seems limited. 
Suppose now that $Y$ is fixed, and let $X$ be any other space. The operations $\Psi_{n(i)}$ and $\tau_{n(i)}{ }^{*}$ then have as domain the group $H^{n(i)}\left(X ; \pi_{i}\right)$, while $\Phi_{n(i-1)-1}$ has this group as range. In $\S 3$ we show that Image $\Phi_{n(i-1)-1} \subset \operatorname{Kernel} \Psi_{n(i)}(i \geqq 1)$. Define, for $i>1$

$$
\mathfrak{N}^{n(i)}(X, Y)=\frac{\text { Kernel } \tau_{n(i)}{ }^{*} \cap\left[\text { Kernel } \Psi_{n(i)}\right]}{\text { Kernel } \tau_{n(i)}{ }^{*} \cap \operatorname{Image} \Phi_{n(i-1)-1}}
$$

(Since $\Psi_{n(i)}$ is not necessarily additive we denote by $\left[\operatorname{Kernel} \Psi_{n(t)}\right]$ the least subgroup of $H^{n(i)}\left(X ; \pi_{i}\right)$ containing $\operatorname{Kernel} \Psi_{n(i)}$.

As above suppose that $Y$ is $n$-simple for all $n \geqq 1$ and suppose in addition that $\pi_{i} \in \mathscr{G}, i \geqq 1$. (These conditions are fulfilled, for example, if the singular homology groups of $Y \in \mathscr{G}$ and either $Y$ is simply-connected or $Y$ is an $H$-space $[17,5]$.) We shall prove

THEOREM 2.2. Let $X$ be a finite-dimensional CW-complex. If $\mathfrak{N}^{n(i)}(X, Y)=0$ for all $i \geqq 2$, then $N[X, Y]=0$.

In $\S 4$ we compute the invariants $\Phi, \Psi$, and $\tau$ for the classifying spaces of the stable classical groups, and then apply Theorem 2.2 to the problem of classifying (stable) vector space bundles over complexes, obtaining as a special case the theorem of Peterson mentioned in $\$ 1$.

REMARK. Theorem 2.2 is only a first level result, in the sense that the groups $\mathfrak{N}^{n(i)}(X, Y)$ are defined using only primary cohomology operations. It is possible to define, for each $n \geqq 1$, a sequence of $n$th order cohomology operations associated with $Y$, and using these to define a sequence of groups with numerator the same as $\mathfrak{N}^{n(i)}(X, Y)$ but with larger denominators. In $\S 3$ we sketch the definition of the second order operations, but for simplicity we do the details of the method only using primary operations.

3. Proof of Theorem 2.2. Suppose that $Y$ is a simply-connected space, and denote by $\Omega Y$ the loop space of $Y$. A Postnikov system for $\Omega Y$ is obtained by applying the loop functor $\Omega$ to all the spaces and maps in diagram (2.1). Denote by $\Omega Y \rightarrow P Y \rightarrow Y$ the path fibre space over $Y[17$, Chapter 4, $\S 4]$. Recall that a MoorePostnikov [13] decomposition of this fibre space gives a sequence of fibre spaces (and commutative diagrams)

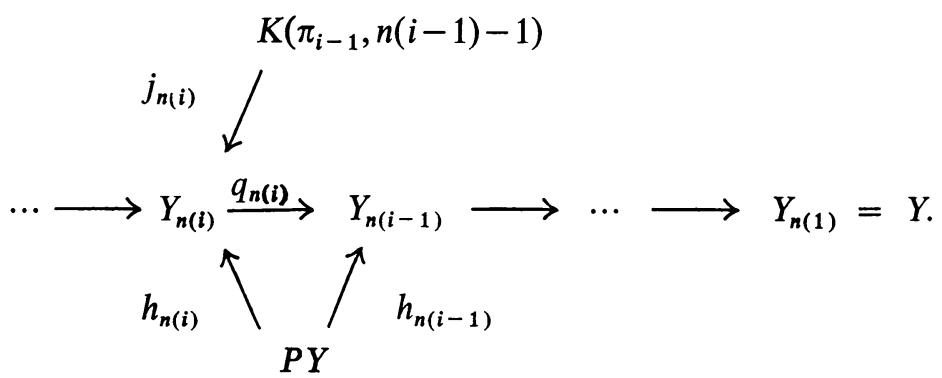


Denote by $p_{n(i)}$ the composite map $Y_{n(i)} \rightarrow \cdots \rightarrow Y$. This is a fibre map and the fibre space $Y_{n(i)} \rightarrow Y$ is induced from the fibre space $P Y^{n(i-1)} \rightarrow Y^{n(i-1)}$ by the map $g_{n(i-1)}$ of $Y$ into $Y^{n(i-1)}$. Thus $p_{n(i)}$ has fibre $\Omega Y^{n(i-1)}$ and $h_{n(i)}$ restricted to $\Omega Y$ is $\Omega g_{n(i-1)}$. Moreover $Y_{n(i)}$ is $(n(i)-1)$-connected and $p_{n(i)}$ induces an isomorphism on homotopy groups in dimensions $\geqq n(i)$. We can construct such a decomposition by the Cartan-Serre-G. Whitehead method of successively killing homotopy groups [7, Chapter 5, §8]. This method is feasible for making computations, since it does not involve the Postnikov system $\left\{Y^{n(i)}\right\}$. (In $\$ 4$ we give two examples of computations using this construction.) By the construction the fibre space $Y_{n(i+1)} \rightarrow Y_{n(i)}$ is induced from the fibre space

$$
\Omega K_{n(i)} \rightarrow P K_{n(i)} \rightarrow K_{n(i)}
$$

$\left(K_{n(i)}=K\left(\pi_{i}, n(i)\right)\right)$ by a map $\gamma_{n(i)}: Y_{n(i)} \rightarrow K_{n(i)}$. Moreover, it is clear that $\gamma_{n(i)}$ is the map $g_{n(1)}$ in the Postnikov system for $Y_{n(i)}$ (see 2.1).

Denote by $\varepsilon_{n(i)}$ and $\iota_{n(i)}$ the respective fundamental classes of $Y_{n(i)}$ and $K_{n(i)}$. That is,

$$
\varepsilon_{n(i)} \in H^{n(i)}\left(Y_{n(i)} ; \pi_{i}\right), \iota_{n(i)} \in H^{n(i)}\left(K_{n(i)} ; \pi_{i}\right) \text { and } \gamma_{n(i)} * \iota_{n(i)}=\varepsilon_{n(i)} .
$$

(We identify $\pi_{i}$ and $\pi_{n(i)}\left(Y_{n(i)}\right)$ by means of $p_{n(i)}{ }^{*}$.) I claim that

$$
\Phi_{n(i)-1}=j_{n(i+1)} * \varepsilon_{n(i+1)}
$$

To see this notice that the inclusion $K\left(\pi_{i}, n(i)-1\right) \subset{ }^{j} Y_{n(i+1)}$ can be factored into

$$
K\left(\pi_{i}, n(i)-1\right) \subset l \Omega Y^{n(i)} \subset{ }^{u} Y_{n(i+1)} .
$$

Let $\tau$ denote the transgression in the fibre space $K\left(\pi_{i+1}, n(i+1)-1\right) \rightarrow Y_{n(i+2)}$ $\rightarrow Y_{n(i+1)}$. Then $\tau\left(\sigma \iota_{n(i+1)}\right)=\varepsilon_{n(i+1)}$, and hence by $\S 4$ of [12], and the naturality of the transgression,

Recall that by [8],

$$
u^{*} \varepsilon_{n(i+1)}=-k_{n(i)-1}(\Omega Y) .
$$

$$
k_{n(i)-1}(\Omega Y)=\sigma k_{n(i)}(Y)
$$

Thus,

$$
j^{*} \varepsilon_{n(i+1)}=l^{*} u^{*} \varepsilon_{n(i+1)}=-l^{*} k_{n(i)-1}(\Omega Y)=-\sigma l^{*} k_{n(i)}(Y)=\sigma \Psi_{n(i)}=\Phi_{n(i)-1}
$$

as claimed. It follows from (3.2) that the invariant $\Phi_{n(i)-1}$ is the same for the space $Y$ and the space $Y_{n(j)}$ for any $j<i$, and the same is then true of the invariant $\Psi_{n(i)}$, provided $\sigma$ is an isomorphism.

Let $f$ be a map from $X$ to $Y_{n(i)}(i \geqq 1)$. We say that $f$ lifts to $Y_{n(j)}(j>i)$, if there is a map $f^{\prime}$ from $X$ to $Y_{n(j)}$ such that $q_{n(i+1)}{ }^{\circ \cdots}{ }^{\circ} q_{n(j)}{ }^{\circ} f^{\prime} \simeq f$. If $X$ is a CW-complex, then $f^{*} \varepsilon_{n(i)}$ is the (single) obstruction to lifting the map $f$ to $Y_{n(i+1)}$.

Recall $[15 ; 19]$ that for any space $X$ the following sequence is exact $(i \geqq 2)$. 


$$
\cdots \rightarrow\left[X, \Omega K_{n(i-1)}\right] \stackrel{j_{n(i) *}}{\longrightarrow}\left[X, Y_{n(i)}\right] \stackrel{q_{n(i) *}}{\longrightarrow}\left[X, Y_{n(i-1)}\right] \stackrel{\gamma_{n(i-1) *}}{\longrightarrow}\left[X, K_{n(i-1)}\right] .
$$

Moreover (see $[16 ; 19]$ ) there is a map

$$
\mu: \Omega K_{n(i-1)} \times Y_{n(i)} \rightarrow Y_{n(i)}
$$

such that for classes $u, v \in\left[X, Y_{n(i)}\right]$

$$
q_{n(i) *}(u)=q_{n(i) *}(v)
$$

if, and only if, there is a class $w \in\left[X, \Omega K_{n(i-1)}\right]$ with $\mu_{*}(w, u)=v$. Using this we prove

LemMa 3.4. Let $f$ be a map from a $\mathrm{CW}$-complex $X$ into $Y_{n(i)}(i \geqq 2)$. The map $q_{n(i)}$ f lifts to $Y_{n(i+1)}$ if, and only if,

$$
f^{*} \varepsilon_{n(i)} \in \operatorname{Image} \Phi_{n(i-1)-1} \subset H^{n(i)}\left(X_{i} ; \pi_{i}\right) \text {. }
$$

Proof. Let $h$ be any map $X \rightarrow Y_{n(i)}$ and let $w \in H^{n(i-1)-1}\left(X ; \pi_{i}\right)$. We regard $w$ as an element of $\left[X, \Omega K_{n(i-1)}\right]$ and set

$$
v=\mu_{*}(w,[h]) \in\left[X, Y_{n(i)}\right] .
$$

Then $q_{n(i) *}(v)=q_{n(i) *}[h]$, and so $q_{n(i)}{ }^{\circ} h^{\prime} \simeq q_{n(i)}{ }^{\circ} h$, where $h^{\prime}$ represents $v$.

Let $\Delta$ denote the diagonal map $X \rightarrow X \times X$. Then $h^{\prime}$ may be taken to be the following composition:

$$
X \stackrel{\Delta}{\rightarrow} X \times X \stackrel{w \times h}{\rightarrow} \Omega K_{n(i-1)} \times Y_{n(i)} \stackrel{\mu}{\rightarrow} Y_{n(i)} .
$$

Moreover the map $\mu$ has the property that if $l_{1}$ and $l_{2}$ denote the respective inclusions $\Omega K_{n(i-1)}, Y_{n(i)} \subset \Omega K_{n(i-1)} \times Y_{n(i)}$, then

$$
\mu \circ l_{1} \simeq j_{n(i)}, \text { and } \mu \circ l_{2} \simeq \text { identity. }
$$

Therefore, since $Y_{n(i)}$ is $(n(i)-1)$-connected, setting $\varepsilon=\varepsilon_{n(i)}$ and $\Phi=\Phi_{n(i-1)-1}$ we obtain

$$
\begin{aligned}
h^{\prime *} \varepsilon & =\Delta^{*} \circ(w \times h)^{*} \mu^{*}(\varepsilon) \\
& =\Delta^{*} \circ\left(w \times h^{*}\left(j_{n(i)}\right)^{*} \varepsilon \otimes 1+1 \otimes \varepsilon\right) \\
& =\Phi(w)+h^{*} \varepsilon .
\end{aligned}
$$

To apply this to the proof of Lemma 3.4, suppose first that there is a class $u \in H^{n(i-1)-1}\left(X ; \pi_{i}\right)$ such that $f^{*} \varepsilon=\Phi(u)$. Take $h=f, w=-u$ and construct $h^{\prime}$ as above. Since $\Phi$ is additive (it is a suspension),

$$
h^{\prime *} \varepsilon=\Phi(-u)+\Phi(u)=-\Phi(u)+\Phi(u)=0,
$$

and so $h^{\prime}$ lifts to $Y_{n(i+1)}$, which provides a lifting of $q_{n(i)} \circ f$ as required.

Conversely, suppose that $g$ is a map from $X$ to $Y_{n(i+1)}$ which lifts $q_{n(i)}{ }^{\circ} f$. Set 
$h=q_{n(i+1)} \circ g$. Since $q_{n(i)} \circ f \simeq q_{n(i)} \circ h$, there is a class $w \in H^{n(i-1)-1}\left(X ; \pi_{i}\right)$ such that $[f]=\mu_{*}(w,[h])$. Take $h^{\prime}=f$. Since $h$ lifts to $Y_{n(i+1)}, h^{*} \varepsilon=0$ and so, by the above equation:

$$
f^{*} \varepsilon=\Phi(w),
$$

which completes the proof of the lemma.

REMARK. By introducing higher order cohomology operations, one can give a necessary and sufficient condition that the map $q_{n(i-\gamma)} \circ \cdots \circ q_{n(i)} \circ f$ lifts to $Y_{n(i+1)}(\gamma>0)$. As an illustration we sketch the case $\gamma=1$. Consider the fibre space $F \stackrel{l}{\rightarrow} Y_{n(i)} \rightarrow Y_{n(i-2)}$, where $p=q_{n(i-1)} \circ q_{n(i)}$. Then, $F=\Omega\left(Y_{n(i-2)}^{n(i+1)}\right)$; that is, a space with two nonvanishing homotopy groups, $\pi_{i-2}$ in dimension $n(i-2)-1$ and $\pi_{i-1}$ in dimension $n(i-1)-1$, and with $\Phi_{n(i-2)-1}$ as $k$-invariant. Set

$$
\Phi_{n(i-2)-1}^{\prime}=\gamma_{n(i)} \circ l: F \rightarrow K_{i} .
$$

This is a secondary cohomology operation, defined on Kernel $\Phi_{n(i-2)-1}$ and taking values in the cosets of Image $\Phi_{n(i-1)-1}$. One then proves an analogous result to Lemma 3.4:

The map $p \circ f$ lifts to $Y_{n(i+1)}$ if, and only if,

$$
f^{*} \varepsilon_{n(i)} \in \operatorname{Image} \Phi_{n(i-1)-1}+\operatorname{Image} \Phi_{n(i-2)-1}^{\prime} \subset H^{n(i)}\left(X ; \pi_{i}\right) .
$$

Suppose now that $X$ is a finite-dimensional CW-complex and that $Y$ is a space whose homotopy groups $\in \mathscr{G}$. Furthermore suppose that $\mathfrak{N}^{n(i)}(X, Y)=0$ for $i \geqq 2$. To prove Theorem 2.2 we must show that if $[f] \in N[X, Y]$, then $f$ is nullhomotopic, and to do this it suffices to show that $f$ can be lifted to each $Y_{n(i)}(i>1)$.

Since $[f] \in N[X, Y], f^{*} \varepsilon_{n(1)}=0$ and hence $f$ lifts to $Y_{n(2)}$. Moreover $Y_{n(2)}$ is simply-connected and so Lemma 3.4 can be applied to the spaces $Y_{n(i)}(i>2)$ lying over $Y_{n(2)}$. Suppose then that for some $i>2, f$ lifts to a map, $f_{i}$, from $X$ to $Y_{n(i)}$. To complete the inductive step we must show that $f$ lifts to $Y_{n(i+1)}$, which will then complete the proof of Theorem 2.2.

Set $u=f_{i}^{*} \varepsilon_{n(i)} \in H^{n(i)}\left(X ; \pi_{i}\right)$. Now $\Psi_{n(i)}$ is the first $k$-invariant for the space $Y_{n(i)}$, and therefore the map $\Psi_{n(i)}{ }^{\circ} \gamma_{n(i)}$ is null-homotopic. Thus Image $\Phi_{n(i-1)-1}$ C Kernel $\Psi_{n(i)}$, since $\Phi_{n(i-1)-1}=j_{n(i)}^{*} \gamma_{n(i)}{ }^{*} \iota_{n(i)}$, by 3.2. Similarly,

$$
\Psi_{n(i)}(u)=\Psi_{n(i)} f_{i}^{*} \varepsilon_{n(i)}=f_{i}^{*} \Psi_{n(i)} \gamma_{(n i)} * \iota_{n(i)}=0,
$$

which shows that $u \in \operatorname{Kernel} \Psi_{n(i)}$.

Consider now the invariant $\tau_{n(i)}$. If $\tau_{n(i)}=0$, then trivially $u \in \operatorname{Kernel} \tau_{n(i)}{ }^{*}$. If $\tau_{n(i)} \neq 0$, then $\pi_{i}$ is cyclic and there is a class $v \in H^{n(i)}\left(Y ; \pi_{i}\right)$ such that $g^{*} v=\tau_{n(i)} s_{n(i)}$, where $g: S^{n(i)} \rightarrow Y$ represents a generator for $\pi_{i}$. Let $g_{n(i)}: S^{n(i)} \rightarrow Y_{n(i)}$ represent a generator for $\pi_{n(i)}\left(Y_{n(i)}\right)$. Since $p_{n(i)}{ }^{*}$ is an isomorphism we may take $g=p_{n(i)} \circ g_{n(i)}$, and thus obtain

$$
p_{n(i)}{ }^{*} v= \pm \tau_{n(i)} \varepsilon_{(n i)},
$$

using the fact that $g_{n(i)}{ }^{*} \varepsilon_{n(i)}= \pm s_{n(i)}$. Therefore, 


$$
\tau_{n(i)} *(u)=\tau_{n(i)}\left(f_{i}^{*} \varepsilon_{n(i)}\right)= \pm f_{i}^{*} p_{n(i)} * v=f^{*} v=0,
$$

since $f \simeq p_{n(i)} \circ f_{i}$ and $[f] \in N[X, Y]$. Thus in either case $u \in \operatorname{Kernel} \tau_{n(i)} *$. By hypothesis $\mathfrak{N}^{n(i)}(X, Y)=0$, and therefore $u \in$ Image $\Phi_{n(i-1)-1}$. Thus by Lemma $3.4, q_{n(i)} \circ f_{i}$ lifts to $Y_{n(i+1)}$ and therefore so does $f$. This completes the inductive step for the proof of Theorem 2.2.

4. Stable vector bundles. Suppose that $Y$ is a $\mathrm{CW}$-complex such that for some positive integer $N, \Omega_{0}^{N} Y$ has the homotopy type of $Y .\left(\Omega_{0}\right.$ denotes the component of the constant loop.) Then it follows from Theorem 3 of [10] that $\Omega^{N} Y_{N+n(i)}$ and $Y_{n(i)}(i \geqq 1)$ have the same homotopy type. Applying (3.3) we obtain, for $i \geqq 1$,

$$
\Psi_{n(i)}=\sigma^{N} \Psi_{N+n(i)}, \quad \Phi_{n(i)-1}=\sigma^{N} \Phi_{N+n(i)-1},
$$

since $\Psi_{N+n(i)}\left(Y_{N+n(1)}\right)=\Psi_{N+n(i)}(Y)$, by the remark following the proof of (3.2).

We apply this to the case of the classifying spaces for the stable classical groups- $O$ and $U$-defined by Bott [3], studying first the space $B_{o}$. (In general $B_{G}$ denotes a classifying space for a group $G$.) Bott shows that $B_{O} \equiv \Omega_{0}^{8} B_{O}$, with the following periodic homotopy groups.

$$
\begin{array}{l|rrrrrrrr}
\gamma \bmod 8 & 1 & 2 & 3 & 4 & 5 & 6 & 7 & 8 \\
\hline \pi_{\gamma}\left(B_{O}\right) & Z_{2} & Z_{2} & 0 & Z & 0 & 0 & 0 & Z
\end{array} .
$$

Thus by (4.1) there are four distinct (stable) values of the $\Psi$ invariants, since $\Psi_{n(i)}=\sigma^{8} \Psi_{n(i)+8}$. We in fact give the values of $\Phi_{n(i)-1}$, for we then obtain $\Psi_{n(i)}$ by desuspending.

THEOREM 4.2. For the space $B_{0}$ the $\Phi$-operations are as follows. For $k \geqq 0$,

$$
\begin{aligned}
& \Phi_{8 k+1}=\delta_{2} S q^{2}: H^{8 k+1}\left(X ; Z_{2}\right) \rightarrow H^{8 k+4}(X ; Z) \\
& \Phi_{8 k+3}= \pm \delta_{3} P_{3}^{1}+\delta_{2} S q^{4}: H^{8 k+3}(X ; Z) \rightarrow H^{8 k+8}(K ; Z) \\
& \Phi_{8 k+7}=S q^{2}: H^{8 k+7}(X ; Z) \rightarrow H^{8 k+9}\left(X ; Z_{2}\right) \\
& \Phi_{8 k+8}=S q^{2}: H^{8 k+8}\left(X ; Z_{2}\right) \rightarrow H^{8 k+10}\left(X ; Z_{2}\right)
\end{aligned}
$$

The $\tau_{n(i)}$ are given by:

$$
\begin{gathered}
\tau_{1}=\tau_{2}=1 ; \tau_{j}=0 \text { for } j>8 \text { and } j \equiv 1,2 \bmod 8 ; \\
\tau_{8 k+4}=2((4 k+1) !), \quad \tau_{8 k+8}=(4 k+3) !, k \geqq 0 .
\end{gathered}
$$

Here $X$ is any space, $S q^{i}$ and $P_{3}^{j}$ denote the respective $\bmod 2$ and $\bmod 3$ Steenrod operators, and $\delta_{n}(n \geqq 2)$ denotes the Bockstein operator associated with the exact coefficient sequence $0 \rightarrow Z \stackrel{\mathfrak{n}}{\rightarrow} Z \rightarrow Z_{n} \rightarrow 0$.

Using the above invariants we can compute the groups $\mathfrak{N}^{n(i)}\left(K, B_{O}\right)$ defined in $\S 2$. By (1.1) and (2.2) we obtain 
THEOREM 4.3. Let $K$ be a finite-dimensional $\mathrm{CW}$-complex and suppose that $\mathfrak{N}^{n(i)}\left(K, B_{o}\right)=0$ for all $i \geqq 2$. Then two (stable) real vector bundles over $K$ are equivalent if, and only if, they have identical Pontryagin and StiefelWhitney characteristic classes.

Here we have used the fact that the cohomology ring of $B_{O}$ is determined by the universal Pontryagin and Stiefel-Whitney classes.

The integers $\tau_{n(i)}$ in 4.2 are given by the Bott divisibility conditions for the Pontryagin classes of real vector bundles over $4 k$-dimensional spheres [4].

To obtain the $\Phi$-invariants for $B_{o}$, take a Moore-Postnikov decomposition for the fibre space $P B_{O} \rightarrow B_{o}$. Set

$$
B(0, n(i))=Y_{n(i)}
$$

where the $Y$ 's are the spaces given in $\S 2$. (Thus $B(0,1)=B_{O}$.) By $(4.1)$ it is sufficient to compute the invariants $\Phi_{n(i)-1}$ for $4 \leqq i \leqq 7$, as the remaining $\Phi$ 's and all the $\Psi$ 's, are then given by desuspending. Now $\Phi_{7}$ and $\Phi_{8}$ take values with $Z_{2}$ as the coefficient group, and

$$
\Phi_{9} \in H^{12}(Z, 9 ; Z) \approx Z_{2} .
$$

Thus these three invariants can be computed by using $\bmod 2$ coefficients. Moreover

$$
\Phi_{11} \in H^{16}(Z, 11 ; Z) \approx Z_{2}+Z_{3},
$$

and therefore we can determine the mod 2 (respectively, mod3) summand of this operation by using $\bmod 2($ respectively, $\bmod 3)$ coefficients.

We first study the mod 2 cohomology of the spaces $(3) B(0, k)$, applying (3.2) to determine the mod 2 component of the $\Phi$-invariants. We begin with the known result [21],

$$
H^{*}(B(0,4))=Z_{2}\left[W_{4}, W_{6}, W_{7}, \cdots\right],
$$

where the $W$ 's are the images of the universal Stiefel-Whitney classes from $B_{o}$ and where we delete those $W_{i}(i \geqq 2)$ such that $i=2^{r}+1, r \geqq 0$.

Consider the fibering $K(Z, 3) \rightarrow B(0,8) \rightarrow B(0,4)$. Using the result of Serre [18] for $H^{*}(Z, 3)$, we apply Lemma 2.1 of [21] to show that

$$
H^{*}(B(0,8))=Z_{2}\left[W_{8}^{\prime}, W_{12}^{\prime}, W_{14}^{\prime}, \cdots\right] \text {, }
$$

where $W_{i}^{\prime}=q_{8}^{*} W_{i}$, and where we delete those $W_{i}^{\prime}(i \geqq 8)$ such that $i=2^{s}+2^{r}+1$, with either $s \geqq r \geqq 1$ or $s>0$ and $r=0$. Moreover by the Wu formula [23],

$$
S q^{i} W_{8}^{\prime}=W_{8+i}^{\prime} \quad(i=4,6,7) .
$$

(3) In Notices Amer. Math. Soc. 9 (1962), 328-329, R.E. Stong states a complete description of the mod 2 cohomology rings of $B(0, k)$ and $B(U, k)$. Added in proof. See Trans. Amer. Math. Soc. 107 (1963), 526-544. 
To compute the invariants $\Phi_{7}, \cdots, \Phi_{11}$ for $B_{0}$ we need the Serre exact sequence

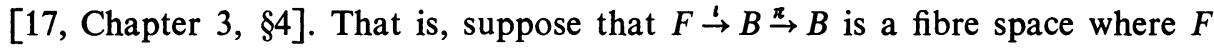
is $(p-1)$-connected and $B$ is $(q-1)$-connected $(p, q \geqq 1)$. Then one has the following exact sequence, using any principal ring for coefficients:

$$
\cdots \rightarrow H^{r-1}(F) \stackrel{\tau}{\rightarrow} H^{r}(B) \stackrel{\pi}{\rightarrow} H^{r}(E) \stackrel{i^{*}}{\rightarrow} H^{r}(F) \rightarrow \cdots \rightarrow H^{p+q-1}(F) .
$$

Here $\tau$ denotes the transgression homomorphism.

In order to compute $\Phi_{7}$ we apply $\left(^{*}\right)$ to the fibering $K(Z, 7) \rightarrow B(0,9) \rightarrow B(0,8)$. For any integral cohomology class $u$ let $\bar{u}$ denote its mod 2 reduction. Then $\tau\left(\bar{\iota}_{7}\right)=W_{8}^{\prime}$ and $\tau\left(S q^{2} \bar{\iota}_{7}\right)=0$, which shows by $\left(^{*}\right)$ and (3.2) that

$$
S q^{2} \bar{\iota}_{7}=j_{9}^{*} \varepsilon_{9}=\Phi_{7} \text {. }
$$

Furthermore,

$$
j_{9}^{*} S q^{2} \varepsilon_{9}=S q^{2} S q^{2} \bar{\iota}_{7}=S q^{3} S q^{1} \bar{\iota}_{7}=0
$$

and hence by $\left({ }^{*}\right)$,

$$
S q^{2} \varepsilon_{9}=0
$$

since $H^{11}(B(0,8))=0$. Notice also that

$$
q_{10}^{*} H^{13}(B(0,9))=0,
$$

since the classes in dimension 13 come from $\varepsilon_{9}$ by squaring operations.

To determine $\Phi_{8}$ we apply (*) to the fibering $K\left(Z_{2}, 8\right) \rightarrow B(0,10) \rightarrow B(0,9)$. Using 4.4 and 4.5 one has that

$$
S q^{2} \iota_{8}=j_{10}^{*} \varepsilon_{10}=\Phi_{8} \text {, and } S q^{3} \varepsilon_{10}=0 .
$$

(The latter fact uses the Adem relation [1] $S q^{3} S q^{2}=0$.) Finally, by the same type of argument, one shows that

$$
S q^{3} \iota_{9}=j_{12}^{*} \bar{\varepsilon}_{12}=\Phi_{9} .
$$

We are left with showing that

$$
S q^{5} \bar{\imath}_{11}=j_{16}^{*} \bar{\varepsilon}_{16}=\bar{\Phi}_{11} \text {. }
$$

Suppose to the contrary that $j_{16}^{*} \bar{\varepsilon}_{16}=0$. We show that this assumption leads to a contradiction. By $\left(^{*}\right)$, applied to the fibering $K(Z, 11) \rightarrow B(0,16) \rightarrow B(0,12)$, we see that $S q^{4} \bar{\varepsilon}_{12} \neq 0$ and that there is a class $u \in H^{16}(B(0,12))$ such that $q_{16}^{*} u=\bar{\varepsilon}_{16}$. Consider the fibering $K\left(Z_{2}, 9\right) \stackrel{j_{12}}{\longrightarrow} B(0,12) \stackrel{q_{12}}{\longrightarrow} B(0,10)$. By [18], $H^{16}\left(Z_{2}, 9\right)$ has the following basis:

$$
S q^{7}\left(\iota_{9}\right), S q^{6} S q^{1}\left(\iota_{9}\right), S q^{5} S q^{2}\left(\iota_{9}\right), S q^{4} S q^{2} S q^{1}\left(\iota_{9}\right) .
$$

Now $j_{12}^{*} \bar{\varepsilon}_{12}=S q^{3} \iota_{9}$, and hence by the Adem relations, 


$$
j_{12}^{*}\left(S q^{4} \bar{\varepsilon}_{12}\right)=S q^{4} S q^{3}\left(\iota_{9}\right)=S q^{5} S q^{2}\left(\iota_{9}\right) .
$$

On the other hand $S q^{7} \varepsilon_{10}, S q^{6} S q^{1} \varepsilon_{10}$ and $S q^{4} S q^{2} S q^{1} \varepsilon_{10}$ are linearly independent in $H^{17}(B(0,10))$. (One sees this by applying $j_{10}^{*}$ to these classes and using the Adem relations in $H^{17}\left(Z_{2}, 8\right)$.) Since $\tau\left(\iota_{9}\right)=\varepsilon_{10}$, it follows that $\tau$ is a monomorphism on a summand complementary to $S q^{5} S q^{2}\left(\iota_{9}\right)$ in $H^{16}\left(Z_{2}, 9\right)$. Thus by $\left(^{*}\right), j_{12}^{*} u$ must belong to the summand spanned by $S q^{5} S q^{2} \iota_{9}$ and hence

$$
u=q_{12}^{*} v+a\left(S q^{4} \varepsilon_{12}\right)
$$

for some class $v \in H^{16}(B(0,10))$.

By a similar argument one shows that

$$
v=q_{10}^{*} w+\left(b S q^{6}+c S q^{4} S q^{2}\right) \varepsilon_{10}
$$

where $w \in H^{16}(B(0,9))$.

Finally, we consider the fibering $K(Z, 7) \rightarrow B(0,9) \rightarrow B(0,8) . \quad H^{16}(Z, 7)$ has $S q^{7} S q^{2} \iota_{7}$ and $S q^{6} S q^{1} S q^{2} \iota_{7}$ as basis elements and these classes transgress to zero in $H^{16}(B(0,8))$. Since $W_{16}^{\prime}$ and $W_{8}^{\prime 2}$ generate $H^{16}(B(0,8))$, we have

$$
w=q_{9}^{*} W_{16}^{\prime}+\left(d S q^{7}+e S q^{6} S q^{1}\right) \varepsilon_{9}
$$

Thus, since $B(0,16)$ is $15-$ connected,

$$
\bar{\varepsilon}_{16}=r^{*} W_{16}^{\prime}
$$

where $r=q_{9} \circ q_{10} \circ q_{12} \circ q_{16}$. But by the Bott divisibility criterion, one has that $W_{16}^{\prime}$ is zero in $B(0,16)$ (see argument by Milnor in [11]) and hence we have obtained a contradiction. Thus,

as claimed.

$$
\Phi_{11}=S q^{5} \bar{\iota}_{11}
$$

This completes the mod 2 calculations needed in 4.2. The remaining calculation is the mod 3 summand in $\Phi_{11}$. Since $\Phi_{3}=\sigma^{8} \Phi_{11}$, it suffices to show that

$$
\pm \delta_{3} P_{3}^{1} \iota_{3}=j_{8}^{*} \varepsilon_{8}
$$

and this follows at once from the fact that $\delta_{3} P_{3}^{1} \iota_{3}$ generates $H^{8}(Z, 3 ; Z)$ and that $H^{*}(B(0,4) ; Z)$ has no 3-torsion. This completes the proof of Theorem 4.2.

We turn now to the classifying space $B_{U}$. Bott [3] shows that $B_{U} \equiv \Omega_{0}^{2} B_{U}$, and that

$$
\pi_{2 i}\left(B_{U}\right)=Z, \quad \pi_{2 i-1}\left(B_{U}\right)=0, \quad i \geqq 1 .
$$

Thus by 4.1 there is just one (stable) $\Phi$ invariant to compute, since $\Phi_{2 i-1}=\sigma^{2} \Phi_{2 k+1}$ $(i \geqq 1)$. (See $\S 3$ of $[14]$.)

THEOREM 4.6. For the space $B_{U}$,

$$
\Phi_{2 i-1}=\delta_{2} S q^{2}: H^{2 i-1}(X ; Z) \rightarrow H^{2 i+2}(X ; Z) \quad(i \geqq 1) .
$$


The $\tau$ invariants are given by

$$
\tau_{2 i}=(i-1) !,
$$

Again we can use these invariants to compute the groups $\mathfrak{N}^{n(i)}\left(X, B_{U}\right)$ (see $\left.\S 2\right)$. Combining (1.1) and (2.1) we obtain

THEOREM 4.7. Let $K$ be a finite-dimensional complex and suppose that $\mathfrak{P}^{n(i)}\left(K, B_{U}\right)=0$ for all $i \geqq 2$. Then two (stable) complex vector bundles over $K$ are equivalent if, and only if, they have identical Chern classes.

This includes Theorem 3.2 of [14]. For if the torsion in $H^{2 i}(K ; Z)$ is relatively prime to $(i-1)$ !, then Kernel $\tau_{2 i}{ }^{*}=0$ and hence $\mathfrak{N}^{2 i}\left(K, B_{U}\right)=0$.

The values of the integers $\tau_{2 i}$ in 4.6 come from the Bott divisibility theorem for complex bundles over spheres [4]. One evaluates $\Phi_{3}$ for $B_{U}$ (hence obtaining all the $\Phi$ 's and $\Psi$ 's) by applying the exact sequence $\left({ }^{*}\right)$ to the fibering $K(Z, 3) \rightarrow B(U, 6) \rightarrow B(U, 4)$. Here $B(U, 2 i)=Y_{2 i}$, where the $Y$ 's are a decomposition for the fibre space $P B_{U} \rightarrow B_{U}$. One uses the fact that $B(U, 2)$ and $B(U, 4)$ have nonzero integral cohomology only in even dimensions. We leave the details to the reader.

REMARK. The classes $\Phi_{n(i)}$ have another interpretation from that given here. They in fact occur as the initial differentials in an exact couple whose spectral sequence converges to $\left[S^{r} X, Y\right\rceil\left(S^{r} X=r\right.$ th suspension of $\left.X, r \geqq 1\right)$. Thus the $\Phi$ 's which we have computed for $B_{0}$ and $B_{U}$ occur in spectral sequences which converge to the $K$-functors

$$
\tilde{K}_{0}^{-r}(X), \tilde{K}_{U}^{-r}(X),
$$

of Atiyah-Hirzebruch [2]. A brief discussion of this topic is given in [22].

5. Appendix. Let $X$ and $Y$ be topological spaces, and for each abelian group $G$ let $\lambda_{G}$ denote the set function defined in $\$ 1$. We define an equivalence relation $\equiv$, between classes in $[X, Y]$ by saying that

$$
[f] \equiv[g] \text { if } \lambda_{G}[f]=\lambda_{G}[g],
$$

for all $G \in \mathscr{G}$. We prove

LEMMA 5.1. Let $X_{i}, Y_{i}(i=1,2)$ be spaces such that $Y_{1}$ and $Y_{2}$ have singular homology groups of finite type. Let $\left[f_{i}\right],\left[g_{i}\right] \in\left[X_{i}, Y_{i}\right](i=1,2)$. If $\left[f_{i}\right] \equiv\left[g_{i}\right]$, then

$$
\left[f_{1} \times f_{2}\right] \equiv\left[g_{1} \times g_{2}\right]
$$

as classes in $\left[X_{1} \times X_{2}, Y_{1} \times Y_{2}\right]$.

Proof. Since each space $Y_{i}$ has homology of finite type, there is a chain complex of finite type, $C_{*}\left(Y_{i}\right)$, which is a chain equivalent subcomplex of the singular complex $S\left(Y_{i}\right)$. Hence for each $G \in \mathscr{G}$, 


$$
H^{*}\left(Y_{i}, G\right) \approx H^{*}\left({ }^{i} C(G)\right),
$$

where ${ }^{i} C(G)=\operatorname{Hom}\left(C_{*}\left(Y_{i}\right), G\right)$, and therefore,

$$
H^{*}\left(Y_{1} \times Y_{2} ; G\right) \approx H^{*}\left({ }^{1} C(G) \otimes{ }^{2} C(G)\right) \text {. }
$$

To show that $\left[f_{1} \times f_{2}\right] \equiv\left[g_{1} \times g_{2}\right]$ it suffices to show that $\lambda_{G}\left[f_{1} \times f_{2}\right]=\lambda_{G}\left[g_{1} \times g_{2}\right]$, where $G$ is the integers and all cyclic groups $Z$, $Z_{p^{r}}, r \geqq 1, p$ a prime. We first show

$$
\lambda_{G}\left[f_{1} \times f_{2}\right]=\lambda_{G}\left[g_{1} \times g_{2}\right], \text { for } G=Z_{p} r, r \geqq 1 .
$$

Suppose that this has been proved for all integers $r$ such that $1 \leqq r<n$. We show that this implies the statement for $r=n$. Let $w \in H^{q}\left({ }^{1} C\left(Z_{p^{n}}\right) \otimes{ }^{2} C\left(Z_{p^{n}}\right)\right)$, $q>0$. By taking canonical bases for the respective cochain complexes $[6$, Chapter $5, \S 8)]$, one can show that

$$
w=\sum_{i} u_{i} \otimes v_{i}+\sum_{1 \leqq j \leqq n-1} \delta_{j}^{\prime}\left(x_{j}\right)
$$

where

$$
u_{i} \in H^{a_{i}}\left({ }^{1} C\left(Z_{p^{n}}\right)\right), v_{i} \in H^{b_{i}}\left({ }^{2} C\left(Z_{p^{n}}\right)\right)\left(a_{i}+b_{i}=q\right),
$$

and

$$
x_{j} \in H^{q-1}\left({ }^{1} C\left(Z_{p^{i}}\right) \otimes{ }^{2} C\left(Z_{p^{i}}\right)\right) .
$$

(Here $\delta_{j}^{\prime}$ is the Bockstein coboundary associated with the exact sequence $0 \rightarrow Z_{p^{n}} \rightarrow Z_{p^{n+j}} \rightarrow Z_{p^{i}} \rightarrow 0$.)

Thus,

$$
\begin{aligned}
\left(f_{1} \times f_{2}\right)^{*} w & =\sum_{i}\left(f_{1} \times f_{2}\right)^{*}\left(u_{i} \otimes v_{i}\right)+\sum_{j} \delta_{j}^{\prime}\left(f_{1} \times f_{2}\right)^{*} x_{j} \\
& =\sum_{i} f_{1}^{*} u_{i} \otimes f_{2}{ }^{*} v_{i}+\sum_{j} \delta_{j}^{\prime}\left(f_{1} \times f_{2}\right)^{*} x_{j} .
\end{aligned}
$$

But $\left[f_{i}\right] \equiv\left[g_{i}\right]$ and therefore,

$$
f_{1}^{*} u_{i}=g_{1}^{*} u_{i}, \quad f_{2}^{*} v_{i}=g_{2}{ }^{*} v_{j}
$$

Moreover by the inductive hypothesis,

$$
\left(f_{1} \times f_{2}\right)^{*} x_{j}=\left(g_{1} \times g_{2}\right) * x_{j} .
$$

Consequently,

$$
\left(f_{1} \times f_{2}\right)^{*} w=\sum_{i} g_{1}^{*} u_{i} \otimes g_{2}{ }^{*} v_{i}+\sum_{j} \delta_{j}^{\prime}\left(g_{1} \times g_{2}\right)^{*} x_{j}=\left(g_{1} \times g_{2}\right)^{*} w,
$$

and thus $\lambda_{G}\left[f_{1} \times f_{2}\right]=\lambda_{G}\left[g_{1} \times g_{2}\right]$, where $G=Z_{p^{n}}$. Since

$$
H^{*}\left({ }^{1} C\left(Z_{p}\right) \otimes{ }^{2} C\left(Z_{p}\right)\right)=H^{*}\left({ }^{1} C\left(Z_{p}\right)\right) \otimes H^{*}\left({ }^{2} C\left(Z_{p}\right)\right),
$$


(5.2) is clearly true for $r=1$, completing the inductive proof. A similar argument shows that $\lambda_{\mathrm{z}}\left[f_{1} \times f_{2}\right]=\lambda_{\mathrm{z}}\left[g_{1} \times g_{2}\right]$. We leave the details to the reader.

Suppose now that $Y$ is a homotopy-associative $H$-space with homology of finite type, and let $\mu: Y \times Y \rightarrow Y$ denote the multiplication. Let $[f],[g] \in[X, Y]$. We define the product of these classes (which we will write as $[f]+[g]$, even though it is not necessarily abelian), as the homotopy class of the map

$$
X \stackrel{\Delta}{\longrightarrow} X \times X \stackrel{f \times g}{\longrightarrow} Y \times Y \stackrel{\mu}{\longrightarrow} Y .
$$

From Lemma 5.1 and the naturality of the set $N[X, Y]$, we have

LEMMA 5.3. If $[f] \equiv\left[f_{1}\right]$ and $[g] \equiv\left[g_{1}\right]$, then $[f]+[g] \equiv\left[f_{1}\right]+\left[g_{1}\right]$.

Let $A$ be any group and suppose that $\sim$ is an equivalence relation on $A$ such that, if $a \sim a_{1}$ and $b \sim b_{1}$ then $a \cdot b \sim a_{1} \cdot b_{1}$. Then the set of equivalence classes, $A / \sim$, has a natural group structure. If $K$ denotes the set of elements in $A$ which are equivalent to the identity of $A$, then $K$ is a normal subgroup of $A$, since it is the kernel of the natural map $A \rightarrow A / \sim$. Moreover, $a \sim b$ if, and only if, $a \cdot b^{-1} \in K$.

Proof of Theorem 1.1. If $X$ is a suspension the proof of the theorem is immediate, since $(f+g)^{*}=f^{*}+g^{*}$. Suppose then that $Y$ is a homotopy-associative $H$-space with homology of finite type. In this case, it need not be true that $(f+g)^{*}=f^{*}+g^{*}$. Let $[f],[g] \in N[X, Y]$. But $[f] \in N[X, Y]$ if, and only if, $[f] \equiv 0$. Thus Theorem 1.1 follows from Lemma 5.3 and the above remarks.

\section{REFERENCES}

1. J. Adem, The relations on Steenrod powers of cohomology classes, Algebraic Topology and Geometry (a symposium in honor of S. Lefschetz), Princeton, N. J., 1956.

2. M. Atiyah and F. Hirzebruch, Vector bundles and homogeneous spaces, Proc. Sympos. Pure Math. Vol. 3, pp. 7-38, Amer. Math. Soc., Providence, R.I., 1961.

3. R. Bott, The stable homotopy of the classical groups, Ann. of Math. (2) 70 (1959), 313-337.

4. - - The space of loops on a Lie group, Michigan Math. J. 5 (1958), 35-61.

5. W. Browder, The cohomology of covering spaces of H-spaces, Bull. Amer. Math. Soc. 65 (1959), 140-141.

6. S. Eilenberg and N. Steenrod, Foundations of algebraic topology, Princeton Univ. Press, Princeton, N. J., 1952.

7. S. Hu, Homotopy theory, Academic Press, New York, 1959.

8. K. Iwata, Note on Postnikov invariants of a loop space, Tôhoku Math. J. 8 (1956), 329-332.

9. I. M. James, On H-spaces and their homotopy groups, Quart. J. Math. 11 (1960), 161-179.

10. J. Milnor, On spaces having the homotopy type of a CW-complex, Trans. Amer. Math. Soc. 90 (1959), 272-280.

11. - Some consequences of a theorem of Bott, Ann. of Math. (2) 68 (1958), 444-449.

12. K. Mizuno, A proof for a theorem of M. Nakaoka, Proc. Japan Acad. 30 (1954), 431-434.

13. J. Moore, Semi-simplicial complexes and Postnikov systems, Proc. Internat. Sympos. on Algebraic Topology, Mexico, 1956. 
14. F. Peterson, Some remarks on Chern classes, Ann. of Math. (2) 69 (1959), 414-420.

15. - - Functionai cohomclogy operations, Trans. Amer. Math. Soc. 86 (1957), 197-211.

16. F. Peterson and E. Thomas, A note on non-stable cohomology operations, Bol. Soc. Mat. Mexicana (2) 3 (1958), 13-18.

17. J.-P. Serre, Homologie singulière des espaces fibrés. Ann. of Math. (2) 54 (1951), 424-505.

18. Cohomologie modulo 2 des complêxes d'Eilenberg-MacLane, Comment. Math. Helv. 27 (1953), 198-232.

19. E. Spanier, Secondary operations on mappings and cohomology, Comment. Math. Helv. 75 (1962), 260-282.

20. N. Steenrod, Cohomology operations and obstructions to extending continuous functions, Lecture notes, Princeton Univ., Princeton, N. J., 1957.

21. E. Thomas, On the cohomology of the classifying space for the stable spinor group., Bol. Soc. Mat. Mexicana (2) 7 (1962), 57-69.

22. - A spectral sequence for $K$-theory, Lecture notes in seminar of $\mathbf{R}$. Bott, Harvard Univ., Cambridge, Mass., 1962.

23. W. Wu, Les i-carrés dans une variété grassmannienne, C. R. Acad. Sci. Paris 230 (1950), 918-920.

UNIVERSITY OF CALIFORNIA,

Berkeley, California 